\title{
Non-conventional Control and Implementation of an Electric Wheelchair Designed to Climb Up Stairs, Controlled via Electromyography and Supported by Artificial Neural Network Processing
}

\author{
Martín L. Guzmán, Juan P. Pinto, Luis F. Reina, and Carlos A. Esquit \\ Department of Electronics Engineering, Del Valle de Guatemala University, \\ Guatemala, Central America \\ \{guz08041,pin08359, Ifreina, caesquit\} @uvg.edu.gt
}

\begin{abstract}
This work is part of a project which main objective is to design and implement an electric wheelchair capable of climbing up stairs. The wheelchair is operated thru silent speech non-conventional control presented on this paper using electromyographic (EMG) signals which are fed to a multiple artificial neural network (ANN) system for pattern recognition. Mechanical design of the wheelchair, powering and sensing aspects are presented on additional papers. Electromyographic signals are captured from the patient's anterior triangle of the neck muscle area using Surface Electromyography (SEMG) with electrodes in bipolar configuration. Silent speech commands from patient's gestural movements of three, four and five phonetically different words are used to interact with a Graphic User Interface (GUI) for wheelchair navigation. The system was tested on five patients, achieving an overall recognition accuracy of $95 \%$ with $0.6 \mathrm{~s}$ maximum response time.
\end{abstract}

Keywords: Artificial Neural Network (ANN), Electromyography (EMG), IIR Digital Filtering, Digital Signal Processing, Discrete Wavelet Transform, Probabilistic Inference, Silent Speech.

\section{Introduction}

Bioelectric signal recognition using Electroencephalography (EEG), Electrocardiography (ECG) and Electromyography (EMG) has allowed the development of interfaces between machines and human's electrical nature. Most of these interfaces have found different applications like robot control and language recognition/translation. Nowadays, one of the main intentions behind developing these kinds of nonconventional interfaces is to allow disabled people, especially paraplegic and quadriplegic people, to control applications by which they can overcome their physical limitations.

In the case of non-conventional interfaces based on Electromyography, some research has been done at the University of Hawaii in association with the NASA Ames Research Center, where researchers developed a sub-auditory speech recognition 
interface using electromyogram sensors [1]. Additional research has been carried out at the Karlsruhe Institute of Technology (KIT), where they have developed a speech recognition system based on myoelectric signals using seven EMG channels [2]. Related work using bioelectric signals was also developed at Del Valle de Guatemala University as part of the ANIMA project [3]. ANIMA implements several non-conventional interfaces, one of them using Electrooculography to control a robot.

Electromyographic signals can be obtained through Surface Electromyography (SEMG) using surface electrodes over the muscles of interest or Needle Electromyography (NEMG) using needles that reach the muscles of interest penetrating the skin. The EMG signals are produced by ion exchange activity in the relaxation and contraction movements of muscle fibers that are activated by motoneurons (central nervous system cells) [4].

To be able to observe the potentials from a specific muscular region in human body (micro-volt scale), a comparison between two electrodes placed at the muscle region of interest and a reference electrode placed at a reference muscle area is needed.

The usable frequency range of surface electromyograhic signals is $20 \mathrm{~Hz}$ to $500 \mathrm{~Hz}$ for most applications. Frequencies outside this range need to be filtered out to keep only the frequencies of interest. Amplification and digitization stages are required in our application in order to send information of the EMG signal (produced by the silent speech gestural movements of the patient) to a computer for further processing.

An artificial neural network is a mathematical or computational model inspired by various aspects of the structure and function of biological neural networks in order to emulate the behavior of the human brain. Artificial neural networks have a great ability to extract patterns from complex and rich data. The adaptive learning, tolerance fault and flexibility are the main advantages behind the idea of using artificial neural networks to perform pattern recognition [5]. On this work a single EMG channel was used to recognize what the patient is trying to say via a silent speech recognition system. For the recognition system multiple feedforward multilayer artificial neural networks were used. Probabilistic inference based on a multiple ANN system was used in order to add robustness to the application.

\section{Experimental Setup}

This work uses silent speech gestural movements from a specific set of phonetically different words in order to generate movement commands for wheelchair navigation. The set is composed of the Spanish words "Eco", "Noviembre", "Omega", "Sigma", "Teléfono" and "Uniforme". The system implemented on this work consists of two main modules: Electromyographic Signal Module and Pattern Recognition Module.

\subsection{Electromyographic Signal Module}

Fig. 1 shows all the necessary stages for electromyographic signal capturing, conditioning and transmission to the Pattern Recognition module for further processing. 


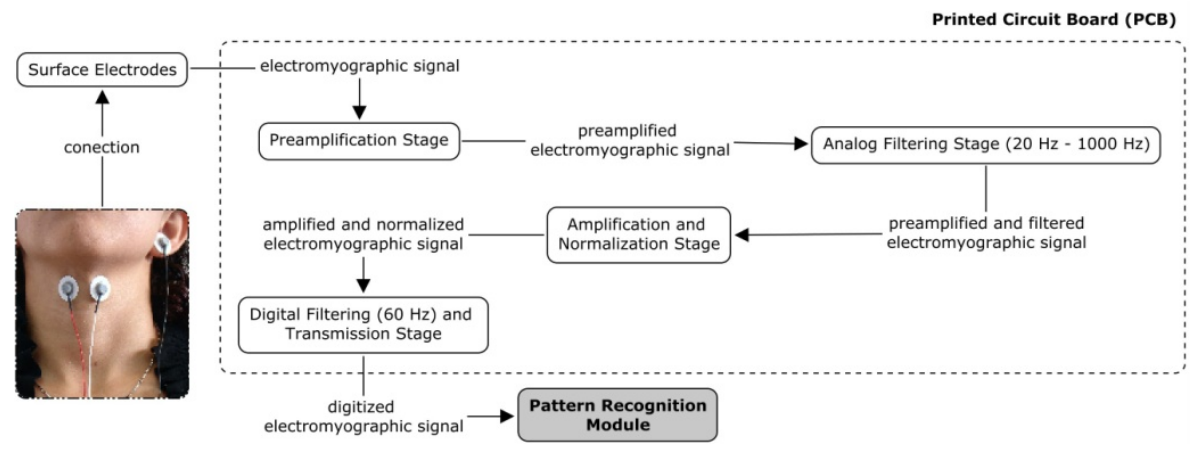

Fig. 1. Electromyographic Signal Module

This module used three $\mathrm{Ag} / \mathrm{AgCl}$ F-E9M-40 surface electrodes (from Grass Technologies) connected to the printed circuit board implemented on this work (Fig. 4). Surface Electromyography was chosen for simplicity and safety. Two surface electrodes were placed in bipolar configuration on the anterior triangle of the neck muscle area and a reference electrode was placed on the left ear lobule (Fig. 2).
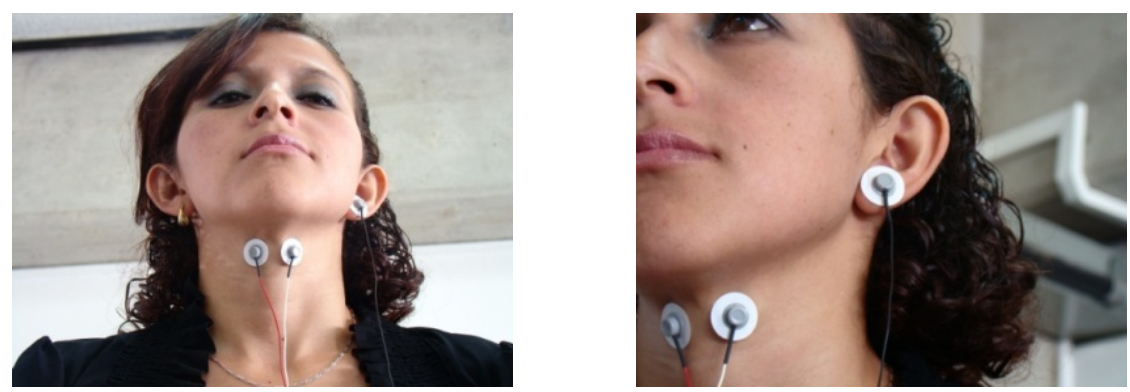

Fig. 2. Surface electrodes placed on the area of interest for electromyographic module

Preamplification Stage. Proper amplitude is needed before any analog or digital signal processing. Instrumental amplifiers offered by different manufacturers are commonly used to achieve this goal. For this stage an AD620 (from Analog Devices) instrumental amplifier was used. This IC offers up to $1000 \mathrm{X}$ adjustable differential gain, high-input impedance and high CMR (Common-Mode Rejection). The gain at this stage is adjusted using a precision potentiometer located on the implemented printed circuit board.

Analog Filtering Stage. Analog filtering ensures that the EMG signal will not contain any noise due to motion artifacts nor any unnecessary frequency components and guarantees a correct sampling for the digitizing stage. This filtering consists of an eight-pole anti-aliasing Butterworth filter with $1000 \mathrm{~Hz}$ cut-off frequency and a 
fourth-pole high-pass Butterworth filter with $20 \mathrm{~Hz}$ cut-off frequency so that the signal's DC component is eliminated. The output of this stage is an EMG signal in the hundreds of mili-volt range and still contaminated by the $60-\mathrm{Hz}$ AC power-line noise.

Amplification and Normalization Stage. Proper amplitude and voltage limits need to be guaranteed prior to digitization. An operational amplifier was used in order to add a DC offset voltage and to amplify the EMG signal. The output of this circuit is an EMG signal centered in the $0-5 \mathrm{~V}$ range so that it is compatible with the digital signal processor's ADC (Analog to Digital Converter). Both the DC offset voltage and the gain are adjusted using precision potentiometers located on the implemented printed circuit board. A voltage limiter circuit built with two high-speed diodes was used to keep the EMG signal in the $0-5 \mathrm{~V}$ range.

Digital Filtering (60-Hz) and Transmission Stage. Digitization and filtering was implemented on a dsPIC30F4013 running at 20 MIPS with $2.6 \mathrm{kHz}$ sampling frequency. Samples serve as input to a difference equation that implements a $60-\mathrm{Hz}$ notch second order IIR digital filter. Two bytes are used to store each 12-bit sample of the EMG signal and a third byte is used as a checksum for the computer to detect corrupt data. The dsPIC microcontroller transfers data to the computer using a UART interface at $115,200 \mathrm{bps}$.

\subsection{Pattern Recognition Module}

Fig. 3 shows all the necessary stages for discretization, detection and recognition of silent speech gestural movements in order to generate commands to be sent to the Wheelchair Control Module (not addressed on this paper).

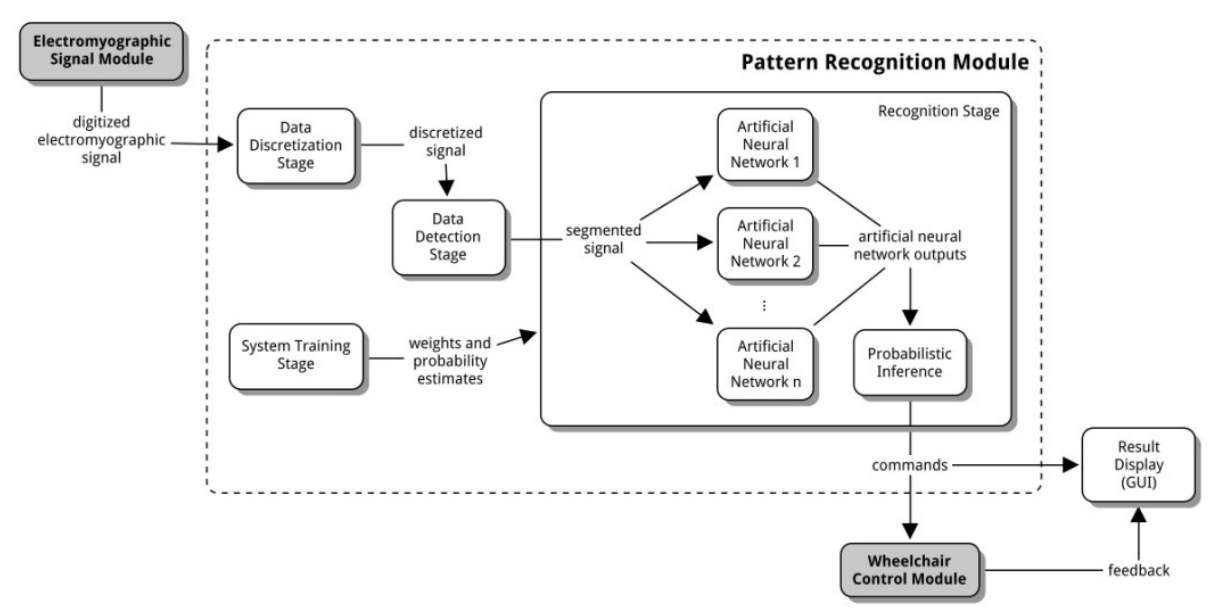

Fig. 3. Pattern Recognition Module 
Discretization Stage. Data discretization is the first step in which the EMG signal is modified once entered into the computer. The stage begins by performing data downscaling for graphical representation purposes and offset adjusting for further processing. Discretization seeks to separate the EMG data and divide it into data blocks in order to accelerate the detection process. On this work the block size was defined as 0.05 seconds of data, which was useful for distinguishing consecutive gestural commands from consecutive syllables within a single gestural command.

Data Detection Stage. Once the signal is divided into a set of blocks, the system performs a data detection method. The aim of this method is to classify all blocks as useful blocks (gestural data present) and not useful blocks (none gestural data present). This data detection method was implemented using confidence intervals based on signal amplitude. These confidence intervals were previously defined by preliminary tests and are calibrated according to the current signal data when the patient is not performing any gestural movements. This stage provides segments (a group of blocks) of a size corresponding to one second (the approximate time it takes a patient to gesticulate a word). Zero-filling is used to complete segments with less of one second of gestural data.

Recognition Stage. This stage receives block segments provided by the data detection stage. These block segments are analyzed by multiple feedforward multilayer artificial neural networks (initial tests were performed using a single feedforward multilayer artificial neural network and final tests were performed using 25 different artificial neural networks). A fifth-order Daubechies Discrete Wavelet Transform was repeatedly applied to the data as a data reduction technique, in order to reduce the amount of data in the EMG signal. The output of this transform was fed into the multiple artificial neural network system. All artificial neural networks design (number of hidden layers and artificial neurons per layer) was based on heuristic methods.

Artificial neural networks provide outputs which are analyzed by a probabilistic inference method based on probability estimates of success and failure of each neural network for each command. This method seeks the probability for a sample to be or not to be a specific command, so the Bayes Theorem was applied to the specific case of a binary partition. The model used to determine the probability for a sample being a specific command is given by (1).

$$
\mathrm{P}\left(C \mid E_{1}, E_{2}, \ldots, E_{n}\right)=\frac{P(C) \prod_{i=1}^{n} P\left(E_{i} \mid C\right)}{P(C) \prod_{i=1}^{n} P\left(E_{i} \mid C\right)+P(\neg C) \prod_{i=1}^{n} P\left(E_{i} \mid \neg C\right)}
$$

Where, $P(C)$ represents the probability that a sample is a command (depends solely on the number of commands) and $P\left(E_{i} \mid C\right)$ represents the observations obtained by the $i$ - $t h$ artificial neural network for the command $C$. These observations consist of a group of independent events. Once the method has computed the probability of each command, a rule indicating the acceptance is carried out to determine whether the sample corresponds to a valid command or to an unidentified command. The rule of acceptance is based on individual probability magnitude. 
System Training Stage. One of the main tasks is the system training. This stage is used to train the artificial neural networks which perform pattern recognition as explained in the previous stage. Supervised learning was chosen, which required the recording of patient samples. System training is personalized, so it was necessary to record samples from all patients individually. Several samples were recorded for each gestural command, between 250 and 400 samples per command depending on the patient's difficulty to produce gestural movements. A session-independent training method as explained in [6] was chosen to obtain robust pattern recognition.

\subsection{Graphic User Interface}

The set of gestural commands is used to interact with a Graphic User Interface (GUI) in order to navigate through a menu of options representing predefined instructions to move the wheelchair. This GUI was implemented using Python language and consists of several screens with either three, four or five options.

The option set allows tasks like rotating the wheelchair at a desired angle and moving forward, backwards and sideways. The option set also allows the use of a camera so the patient can select a destination point for the wheelchair to move with obstacle avoidance (camera navigation algorithm not presented on this paper).

\section{Results}

Fig. 4 shows the implemented printed circuit board for the Electromyographic Signal Module. All the important components and terminals are properly identified over the PCB image.

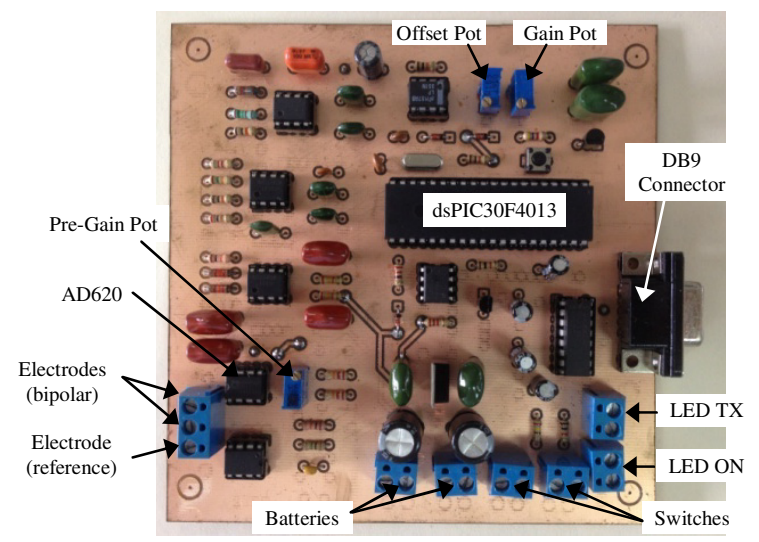

Fig. 4. Electromyographic Signal printed circuit board

Fig. 5 shows oscilloscope images for electromyographic signals after the preamplification stage (in blue) and the normalization and amplification stage (in yellow) for two different gestural commands: "Eco" (left image) and "Noviembre" (right image). 

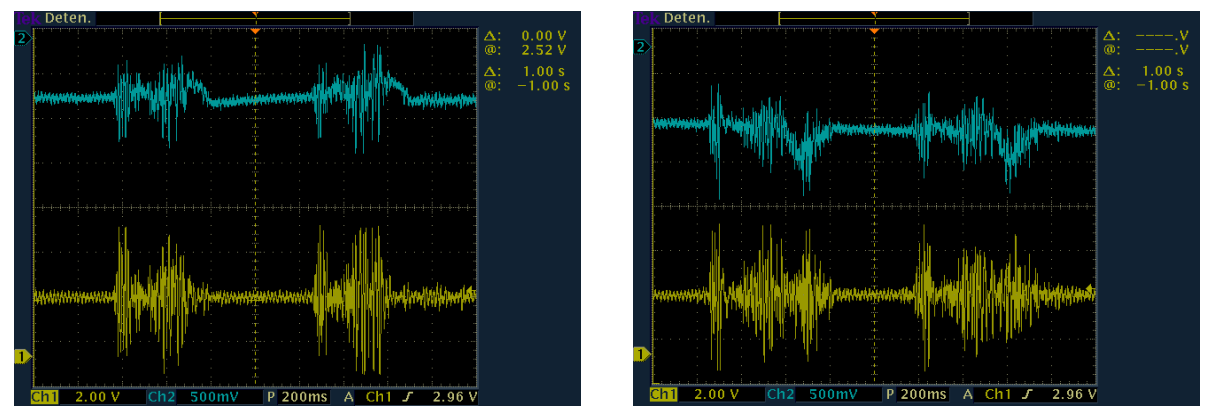

Fig. 5. Electromyographic signals for "Eco" (left) and "Noviembre" (right)

The transfer function in $\mathrm{z}$-domain that describes the $60-\mathrm{Hz}$ notch second order IIR digital filter implemented as a difference equation in a dsPIC microcontroller is given by (2).

$$
H(z)=\frac{0.96436171-1.90848416 z^{-1}+0.96436171 z^{-2}}{1-1.90848416 z^{-1}+0.92872341 z^{-2}}
$$

Fig. 6 and Fig. 7 show examples of sampled Spanish words "Eco", "Sigma", "Noviembre" and "Omega". These samples are the result of the data detection stage.
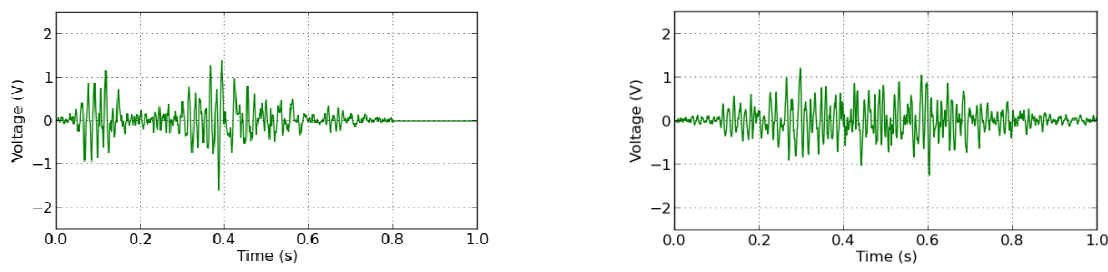

Fig. 6. Silent speech for the words "Eco" (left) and "Sigma" (right)
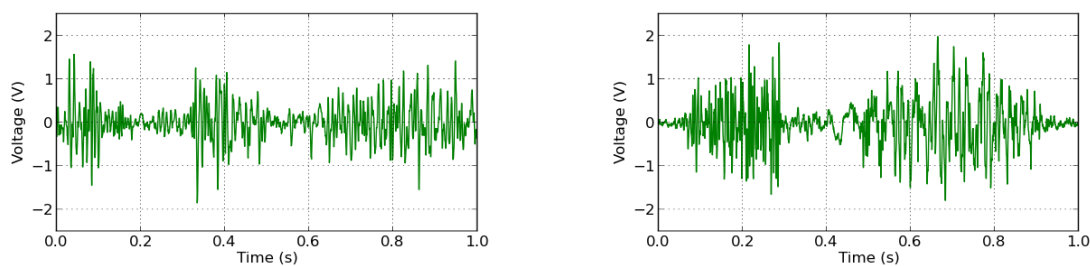

Fig. 7. Silent speech for the words "Noviembre" (left) and "Omega" (right) 
Fig. 8 shows a 10-second silent speech recording in which the patient gestured the Spanish words "Uniforme", "Eco", "Teléfono", "Sigma" and "Noviembre". The data detection line was plotted (in red) alongside the EMG signal (in green) with a positive value (always a $1 \mathrm{~V}$ value) for useful data and a negative value for not useful data (always a $-1 \mathrm{~V}$ value).

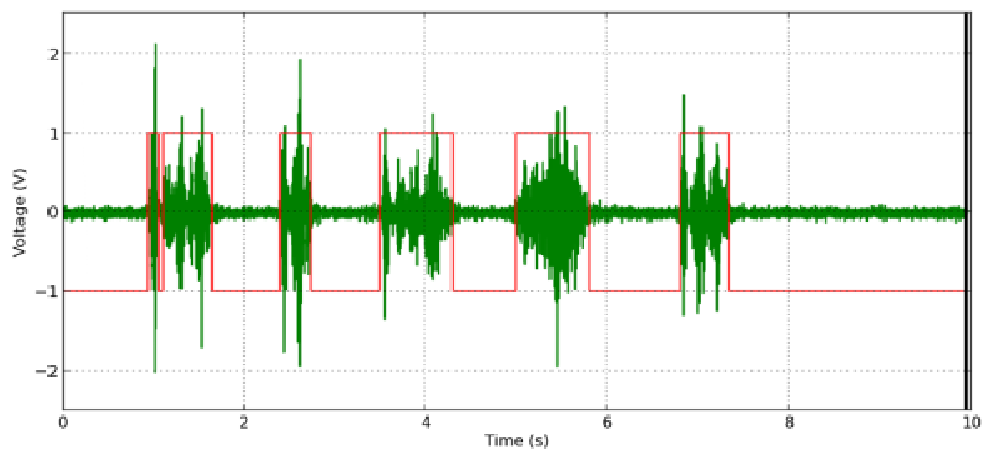

Fig. 8. Silent speech for the Spanish words "Uniforme", "Eco", "Teléfono", "Sigma" and "Noviembre"

Table 1 shows a summary of the results obtained for four different gestural commands. The system was tested on five patients. The worst-case response time for the ANN gestural pattern recognition was 0.6 seconds. The ANN system was programmed on Python and tested on a 32-bit architecture 4GB RAM Intel Core 2 Duo 1.5 GHz processor running Ubuntu, Linux. Single ANN system showed $86 \%$ of accuracy (recognized). Results on Table 1 correspond to the final multiple ANN system with probabilistic inference.

Table 1. Summary of gestural command recognition for all tested patients

\begin{tabular}{ccccc}
\hline Patient & Recognized & Misrecognized & Unrecognized & $\begin{array}{c}\text { Number of } \\
\text { tests }\end{array}$ \\
\hline $\mathbf{1}$ & $94.17 \%$ & $3.33 \%$ & $2.50 \%$ & 120 \\
$\mathbf{2}$ & $97.50 \%$ & $1.67 \%$ & $0.83 \%$ & 120 \\
$\mathbf{3}$ & $95.00 \%$ & $2.50 \%$ & $2.50 \%$ & 120 \\
$\mathbf{4}$ & $93.33 \%$ & $3.33 \%$ & $3.33 \%$ & 120 \\
$\mathbf{5}$ & $95.00 \%$ & $0.83 \%$ & $4.17 \%$ & 120 \\
\hline \hline \multirow{2}{*}{ Average } & $\mathbf{9 5 . 0 0 \%}$ & $\mathbf{2 . 3 3 \%}$ & $\mathbf{2 . 6 7 \%}$ & \\
\hline
\end{tabular}

Fig. 9 shows the GUI implemented on Python so that the patient can interact with the wheelchair by gestural commands. Fig. 10 shows one of the patients while testing the system. 

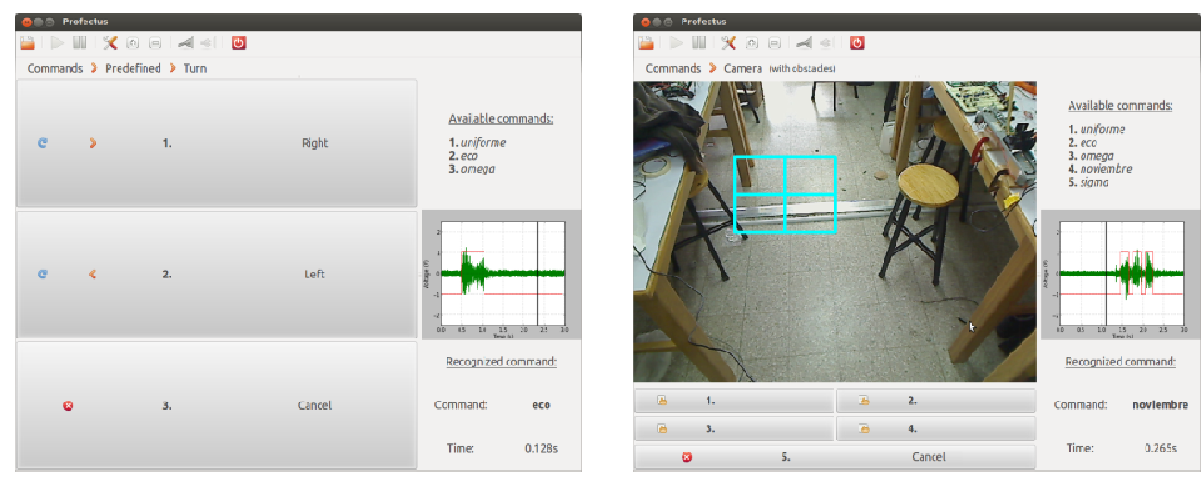

Fig. 9. Graphic User Interface for interaction between patient and wheelchair
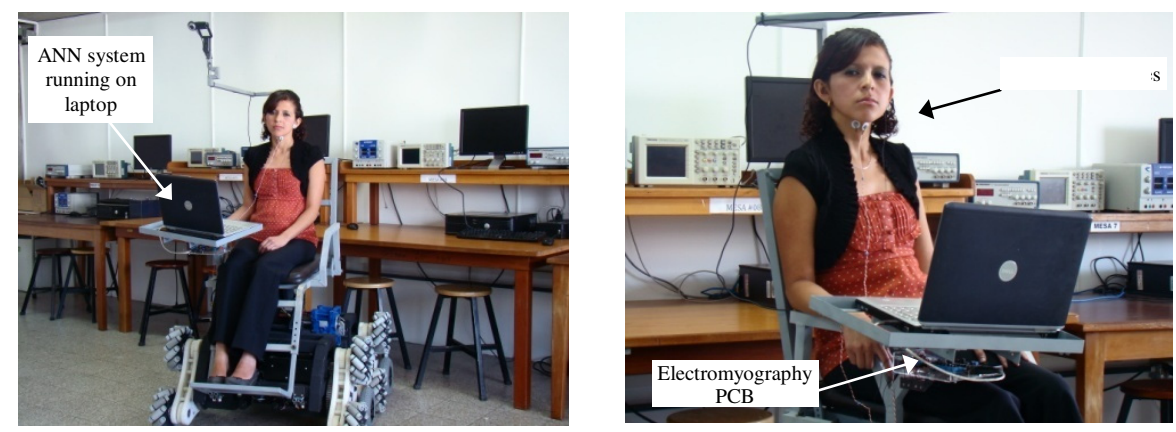

Fig. 10. Electric wheelchair using the implemented non-conventional control

\section{Conclusions}

A system capable of acquiring electromyographic signals from five different subject's gestural movements and recognizing a specific set of words using artificial neural networks was successfully achieved with an accuracy of $95 \%$ for four commands. Tests for three and five commands showed accuracy over 96\%. All tests showed a 0.6-second maximum response time.

Tests showed that location and separation of surface electrodes play an important role on proper electromyography signal capturing.

IIR filtering was chosen over FIR filtering due to its fewer required coefficients which implies faster processing and/or the use of additional channels on the same hardware.

Repetitive use of Wavelet Transform applied to electromyographic data showed to be useful at achieving high accuracy while speeding up the whole ANN system.

The use of multiple artificial neural networks and probabilistic inference offered an average $9 \%$ improvement on accuracy from the original $86 \%$ for single artificial neural network system. 


\section{Future Work}

Future research has to be done to test the system on handicapped subjects (paraplegic and quadriplegic people). Additional gestural commands can be tested so that the system might be useful for more complex interaction applications. Analyzing the possibility for migrating the ANN system running on the laptop to an FPGA-based system would be of interest in terms of cost, area, weight, response time and portability. The Electromyographic Signal Module could be redesigned to include additional signal channels so that there would be more useful data for further processing.

Satisfactory results were obtained for a small vocabulary as used on this work, but exploring similar systems using other approaches like HMM or SVM for EMG pattern recognition would be interesting in order to evaluate the performance for extensive vocabularies in the case of other applications which may require much more than a few words.

Genetic algorithms can be used instead of heuristic methods for ANN design in order to evaluate possible accuracy improvement.

\section{References}

1. Jorgensen, C., Binsted, K.: Sub-Auditory Speech Recognition (2006),

http: //www2 .hawaii.edu/ binsted/papers /

BinstedJorgensenHICSS2006.pdf

2. Maier-Hein, L., Metze, F., Schultz, T., Waibel, A.: Session Independent Non-Audible Speech Recognition using Surface Electromyography (2006),

http://mbi.dkfz-heidelberg.de/mbi/TR/Papers/P14-05.pdf

3. Valdeavellano Wurmser, M.: ANIMA: Métodos no convencionales de interfaz en el control de robots a través de la electroencefalografía y la electrooculografía: Módulo Ocular. Del Valle de Guatemala University, Guatemala (2009)

4. Konrad, P.: The ABC of EMG: A Practical Introduction to Kinesiological Electromyography (April 2005), http: / / demotu .org/aulas / controle/ABCofEMG.pdf

5. Gestal Pose, M.: Introducción a las Redes de Neuronas Artificiales, http://sabia.tic.udc.es/mgestal/cv/RNATutorial/ TutorialRNA.pdf

6. Wand, M., Schultz, T.: Session Independent EMG-Based Speech Recognition (2011), http://csl.anthropomatik.kit.edu/downloads / WandSchultz_Biosignals2011.pdf 